\title{
Cyanide Toxicity to Burkholderia cenocepacia Is Modulated by Polymicrobial Communities and Environmental Factors
}

\author{
Steve P. Bernier ${ }^{1 *}$, Matthew L. Workentine ${ }^{1 \dagger}$, Xiang $L^{2}{ }^{2}$, Nathan A. Magarvey ${ }^{2}$, \\ George A. O'Toole ${ }^{3}$ and Michael G. Surette ${ }^{1,2 *}$
}

OPEN ACCESS

Edited by:

Yuji Morita

Aichi Gakuin University, Japan

Reviewed by:

Eric Déziel,

Institut National de la Recherche

Scientifique, Canada

Sascha Brunke,

Hans-Knöll-Institut, Germany

Haider Abdul-Lateef Mousa,

University of Basrah, Iraq

Rolf Kümmerli,

University of Zurich, Switzerland

*Correspondence:

Steve P. Bernier sbernier19@gmail.com;

Michael G. Surette

surette@mcmaster.ca

${ }^{\dagger}$ Present Address:

Matthew L. Workentine, Faculty of Veterinary, University of

Calgary, Calgary, Canada

Specialty section: This article was submitted to

Infectious Diseases,

a section of the journal

Frontiers in Microbiology

Received: 16 January 2016 Accepted: 02 May 2016

Published: 18 May 2016

Citation:

Bernier SP, Workentine ML, Li X

Magarvey NA, O'Toole GA and

Surette MG (2016) Cyanide Toxicity to

Burkholderia cenocepacia Is

Modulated by Polymicrobial

Communities and Environmental Factors. Front. Microbiol. 7:725. doi: 10.3389/fmich.2016.00725

\begin{abstract}
1 Department of Medicine, Faculty of Health Sciences, Farncombe Family Digestive Health Research Institute, McMaster University, Hamilton, ON, Canada, ${ }^{2}$ Department of Biochemistry and Biomedical Sciences, Faculty of Health Sciences, McMaster University, Hamilton, ON, Canada, ${ }^{3}$ Department of Microbiology and Immunology, Geisel School of Medicine at Dartmouth, Hanover, NH, USA
\end{abstract}

Microbes within polymicrobial communities can establish positive and negative interactions that have the potential to influence the overall behavior of the community. Pseudomonas aeruginosa and species of the Burkholderia cepacia complex (Bcc) can co-exist in the lower airways, however several studies have shown that $P$. aeruginosa can effectively kill the Bcc in vitro, for which hydrogen cyanide $(\mathrm{HCN})$ was recently proposed to play a critical role. Here we show that modification of the environment (i.e., culture medium), long-term genetic adaptation of $P$. aeruginosa to the cystic fibrosis (CF) lung, or the addition of another bacterial species to the community can alter the sensitivity of Burkholderia cenocepacia to $P$. aeruginosa toxins. We specifically demonstrate that undefined rich media leads to higher susceptibility of $B$. cenocepacia to $P$. aeruginosa toxins like cyanide as compared to a synthetic medium (SCFM), that mimics the CF lung nutritional content. Overall, our study shows that the polymicrobial environment can have profound effects on negative interactions mediated by $P$. aeruginosa against $B$. cenocepacia. In fact, evolved $P$. aeruginosa or the presence of other species such as Staphylococcus aureus can directly abolish the direct competition mediated by cyanide and consequently maintaining a higher level of species diversity within the community.

Keywords: competition, cyanide, polymicrobial, community, metabolites, interactions

\section{INTRODUCTION}

Microbes rarely live alone in their natural environments but typically grow as part of multispecies communities (Curtis et al., 2002; Hall-Stoodley et al., 2004), an observation first made by Louis Pasteur (Pasteur and Joubert, 1877). This scenario is also true of infections, the majority of which are now recognized as polymicrobial (Peters et al., 2012). The long-term development of chronic polymicrobial infections in the lower airways is a common feature of obstructive lung diseases such as cystic fibrosis (CF), chronic pulmonary obstructive disease (COPD), and asthma (Huang and Lynch, 2011; Han et al., 2012; Dickson et al., 2014; Surette, 2014). Polymicrobial communities made of Pseudomonas aeruginosa and representative bacterial species from the non-pathogenic normal microbiota showed enhanced disease severity in different model systems compared to 
their monospecies counterparts (Duan et al., 2003; Sibley et al., 2008; Korgaonkar et al., 2013) demonstrating that interspecies interactions play a key role in disease progression. Co-infection by multiple primary pathogens is also common in the lower airways where bacterial pathogens like P. aeruginosa, Staphylococcus aureus, Burkholderia cepacia complex (Bcc), Stenotrophomonas maltophilia, Streptococcus milleri/anginosus group, and Haemophilus influenzae can co-exist (Harrison, 2007; Lipuma, 2010; Han et al., 2012; Surette, 2014).

Mixed $P$. aeruginosa and Bcc infections have been described in the lower airways of patients suffering from CF (Jacques et al., 1998; Lambiase et al., 2006; Schwab et al., 2014) and COPD (Han et al., 2012), wherein disease severity was reported to be worse than mono-infections in both humans (Jacques et al., 1998) and mice (Bragonzi et al., 2012) suggesting in vivo synergistic interactions or additive effects. Despite the fact that both pathogens can co-exist in the lower airways, several studies have reported that $P$. aeruginosa outcompetes and/or kills species of the Bcc both in vitro (Tomlin et al., 2001; AlBakri et al., 2004; Bakkal et al., 2010; Bragonzi et al., 2012; Costello et al., 2014; Rüger et al., 2014; Schwab et al., 2014; Smalley et al., 2015) and in a chronic lung infection mouse model (Bragonzi et al., 2012). Consequently, these observations strongly suggest that in mixed communities the fitness of $P$. aeruginosa is greater than Burkholderia, which may play a critical role in co-infections in terms of disease progression (Bragonzi et al., 2012). In vitro studies have suggested that pyocins, phenazines, rhamnolipids, hydrogen cyanide (HCN), the siderophore pyoverdine and other molecules yet to be identified are released by $P$. aeruginosa as a multifactorial strategy to outcompete or kill Bcc species (Tomlin et al., 2001; Bakkal et al., 2010; Costello et al., 2014; Smalley et al., 2015). However, co-infections for which Bcc species are numerically dominant over P. aeruginosa in the CF lungs (Schwab et al., 2014; Carmody et al., 2015) would argue against a model where Bcc species are always outcompeted by Pseudomonas. In fact, in vitro cocultures with Burkholderia multivorans showed that anaerobic growth reduced the competitive advantage of $P$. aeruginosa and consequently increased Burkholderia survival compared to aerobic conditions (Schwab et al., 2014). The latter supported the hypothesis that environmental conditions might impact competitive interactions between $P$. aeruginosa and the $\mathrm{Bcc}$ by affecting Pseudomonas toxicity, Burkholderia tolerance, or both. Consequently, to better appreciate the in vivo dynamics of mixed $P$. aeruginosa and Bcc infections and their long-term consequences on disease progression, we must improve our in vitro molecular understanding of microbial interactions in the context of polymicrobial communities, including competition.

In the current study, we aim to expand our understanding of competitive interactions in mixed communities of $P$. aeruginosa and species of the Bcc. Herein, we first demonstrate that environmental factor(s) (i.e., growth medium) influence the competitive advantage of $P$. aeruginosa over Burkholderia cenocepacia (strain K56-2) in well-shaken aerobic co-cultures. More specifically, we show that the tolerance of $B$. cenocepacia K56-2 to cyanide and $P$. aeruginosa-derived metabolites is strongly affected by growth medium for which $B$. cenocepacia is more susceptible in rich media compared to chemically defined media. In addition to environmental factors, we show that the long-term adaptation of $P$. aeruginosa to the CF lung also has an impact on its toxicity where half of the $P$. aeruginosa isolates tested in co-culture pairs were dominated by $B$. cenocepacia within $24 \mathrm{~h}$. However, although $\mathrm{HCN}$-mediated toxicity is highly potent against the $B c c$, it is not alone sufficient for Burkholderia killing in co-cultures as the less toxic quorum sensing (QS) Pseudomonas mutants $\triangle l a s R$ and $\triangle p q s A$ and all tested $P$. aeruginosa $\mathrm{CF}$ adapted isolates were positive for the production of $\mathrm{HCN}$ but did not show complete killing, supporting a multifactorial killing model. Lastly, we show that cocultures with cyanide-tolerant bacteria such as $S$. aureus and the less toxic QS rhlR mutant of P. aeruginosa allowed B. cenocepacia to survive and even grow at inhibitory concentrations of cyanide via the release of heat labile molecules(s) with $S$. aureus being the most protective.

\section{MATERIALS AND METHODS}

\section{Bacterial Strains, Plasmids, Chemicals and General Growth Conditions}

Bacterial strains and plasmids used in this study are described in Table S1. Bacterial strains were routinely grown in Lysogeny broth (LB; Miller recipe) or on $1.5 \%$ LB-Miller agar (EMD Chemicals Inc., Gibbstown, NJ, USA) and incubated at $37^{\circ} \mathrm{C}$. BHI (Becton, Dickinson and Company [BD], Sparks, MD, USA), SCFM (Palmer et al., 2007), and M9 minimal medium (BD) containing $0.5 \%$ glucose $\left(\mathrm{M}_{\mathrm{Glc}}\right)$ or $0.5 \%$ casamino acids $\left(\mathrm{M} 9_{\mathrm{CAA}}\right.$; $\left.[\mathrm{BD}]\right)$ were also used where specified in the text. BHI and $\mathrm{LB}$ media were diluted with water to make them less rich in nutrients $(1 / 4$ or $1 / 10)$. Antibiotics were supplemented to the culture medium of $P$. aeruginosa strains when appropriate at the following concentrations: tetracycline (Tet), $150 \mu \mathrm{g} \mathrm{ml}^{-1}$; gentamicin (Gm), $50 \mathrm{\mu g} \mathrm{ml}^{-1}$; trimethoprim (Tp), $100 \mu \mathrm{g} \mathrm{ml}^{-1}$; ceftazidime (Caz), $32 \mu \mathrm{g} \mathrm{ml}^{-1}$; and ciprofloxacin (Cpx), $5 \mu \mathrm{g}$ $\mathrm{ml}^{-1}$. Phenazine-1-carboxylic acid (PCA) was purchased from Princeton BioMolecular Research (Princeton, NJ, USA; Catalog no. PBMR030094) and dissolved in dimethyl sulfoxide (DMSO) at a final concentration of $2 \mathrm{mg} \mathrm{ml}^{-1}$. Pyocyanin was purchased from Sigma-Aldrich (St. Louis, MO, USA; Catalog no. P0046) and dissolved in DMSO at a final concentration of $10 \mathrm{mg} \mathrm{ml}^{-1}$. Potassium cyanide (KCN) was purchased from Sigma-Aldrich (Catalog no. 11813). Stock solutions of KCN (1 M in water) were diluted to $250 \mathrm{mM}$ using $1 \mathrm{M}$ MOPS buffered to $\mathrm{pH} 7.0$ and filter sterilized using $0.2 \mu \mathrm{m}$ filters (Millipore, Billerica, MA, USA). All other chemicals used in this study were obtained from Sigma-Aldrich.

\section{Monoculture and Co-Culture Growth, Extraction of Spent Medium, and Bacterial Survival Quantification}

Bacterial cells from overnight cultures ( $4 \mathrm{ml}$ of LB medium in borosilicate glass test tubes) were spun down ( $2 \mathrm{~min}, 8000 \mathrm{rpm})$ and washed once in phosphate buffered saline (PBS) and used for inoculating liquid cultures in shaken flasks. (i) Monoculture and 
co-culture (flasks). Flasks containing $25 \mathrm{ml}$ of culture medium were inoculated with $80 \mu \mathrm{l}$ of washed cells (1:300 dilution) and incubated for $24 \mathrm{~h}$ at $37^{\circ} \mathrm{C}$ with shaking (175 rpm). (ii) Bacterial survival quantification. Viable cell counts were used to determine bacterial growth or survival of both monoculture and co-culture growth. Bacterial cultures were serially diluted and plated on agar-containing medium with a detection limit of 200 colony forming units (CFUs) $\mathrm{ml}^{-1}$. For mixed cultures, selective agar media and colony morphotypes were used for accurate CFU counts for each bacterial species. To accurately quantify Bcc viable counts in co-cultures, $\mathrm{LB}$ agar supplemented with $\mathrm{Gm}$ $\left(50 \mu \mathrm{g} \mathrm{ml}^{-1}\right)$ and/or Columbia naladixic acid (CNA) agar plates were used. CFU counts for $P$. aeruginosa PA14 derivative strains were directly determined by colony morphotype on non-selective agar plates since their colonies are bigger and shiny compared to those from B. cenocepacia K56-2 that are smaller and rough (Bernier et al., 2008). To determine viable CFUs of $P$. aeruginosa clinical isolates in co-cultures, a combination of selective LB agar containing Caz $\left(32 \mu \mathrm{g} \mathrm{ml}^{-1}\right)$, Cpx $\left(5 \mu \mathrm{g} \mathrm{ml}^{-1}\right)$ or Tp $(100 \mu \mathrm{g}$ $\mathrm{ml}^{-1}$ ) in addition to $\mathrm{Gm}$ and CNA agar plates were used. (iii) Extraction of bacterial spent media. Bacterial cells from 25-ml cultures in flasks $(24 \mathrm{~h})$ were spun down and supernatant was filtered using $0.2 \mu \mathrm{m}$ filters (Millipore) and used immediately or stored at $4^{\circ} \mathrm{C}$. (iv) Monoculture growth in 96-well plates. Overnight cultures were diluted to an $\mathrm{OD}_{600}$ of 0.1 and $0.5 \mu \mathrm{l}$ of the diluted culture was added to $100 \mu \mathrm{l}$ of culture medium per well (1:200 dilution) in standard 96-well microtiter dish plate and incubated $37^{\circ} \mathrm{C}$ for $24 \mathrm{~h}$ with constant shaking in a plate reader (Synergy ${ }^{\mathrm{TM}} \mathrm{H} 1$, BioTek $^{\circledR}$ Instruments, Inc., Winooski, VT) and optical density measurements $\left(\mathrm{OD}_{600}\right)$ were taken every $30 \mathrm{~min}$. To avoid evaporation, $65 \mu \mathrm{l}$ of filtered mineral oil was added on top of the bacterial culture in each well.

\section{Determination of the Minimal Inhibitory Concentration (MIC) and the Fractional Inhibitory Concentration Index (FICl)}

MIC values of small molecules and spent media were determined by macrodilutions in LB as previously described (Hacek et al., 1999) with minor modifications. FICI values were determined as previously described (Odds, 2003; Pillai et al., 2005). Briefly, overnight cultures were diluted to an $\mathrm{OD}_{600}$ of 0.1 and added to 96-well plates at a final dilution of 1:200 (v/v). The final volume per well was $100 \mu \mathrm{l}$ including the test compound and/or supernatant serially diluted. Inoculated plates were sealed with a sticky breathable membrane (Breathe-Easy, USA Scientific, Cat No. 9123-6100) and incubated for $24 \mathrm{~h}$ at $37^{\circ} \mathrm{C}$ with shaking (175 rpm).

\section{Genetic Manipulations}

Plasmids constructed in this study (Table S1) were made using homologous recombination in Saccharomyces cerevisiae as previously described (Shanks et al., 2006). Restriction enzymes and Phusion High-Fidelity DNA polymerase were obtained from New England BioLabs (Ipswich, MA, USA). All newly constructed plasmids were sequenced (MOBIX Lab, McMaster University, Hamilton, ON) using the universal primers M13 forward and reverse for verification (i) Deletion of hcnABC in $P$. aeruginosa. Briefly, two discontinuous fragments with overlapping sequences from primer design were PCR amplified with the primer pairs hcn1-5L hcn13 and hon2-5 hon2-3L (Table S1). The PCR fragments were subsequently cloned into plasmid pMQ30 (linearized with SmaI) via homologous recombination in $S$. cerevisiae. The resulting plasmid pMQ30hcnKO was transformed into E. coli SM10 and used to conjugate $P$. aeruginosa for $\sim 6 \mathrm{~h}$ on LB agar and transconjugants were selected on Pseudomonas Isolation Agar (PIA; BD) containing $\mathrm{Gm}$ at $100 \mu \mathrm{g} \mathrm{ml}{ }^{-1}$. Excision of the plasmid was performed onto $\mathrm{LB}$ agar (no $\mathrm{NaCl}$ ) containing 5 $\%$ sucrose $(\mathrm{w} / \mathrm{v})$ at $30^{\circ} \mathrm{C}$. Confirmation of the mutant genotype was performed by PCR using the primers henV5 and henV3 (Table S1) on $\mathrm{Gm}^{S}$ clones. (ii) Construction of an HCN-expressing vector. To build a plasmid expressing $\mathrm{HCN}$, we amplified by PCR hcn $A B C$ from $P$. aeruginosa PA14 using primers henABC-5L and hcnABC-3L (Table S1). This fragment was cloned into SmaIdigested PMQ72 via homologous recombination in S. cerevisiae. This vector harbors the arabinose-inducible promoter system of E. coli $\left(P_{B A D^{-}-a r a C}\right)$, therefore addition of arabinose $(0.1-0.4 \%)$ allows the induction of the $h c n A B C$ operon and production of $\mathrm{HCN}$.

\section{Detection of Volatile Hydrogen Cyanide and Rhamnolipids}

(i) Hydrogen cyanide. Detection of $\mathrm{HCN}$ was performed as previously described (Castric and Castric, 1983) with minor modifications. Briefly, overnight cultures were transferred into 384-well plates containing $75 \mu \mathrm{l}$ of LB per well using pin replicator (VP408; vol. $0.2 \mu \mathrm{l}-\mathrm{V} \& \mathrm{P}$ Scientific, San Diego, CA, USA). The inoculated 384-well plates were then covered with dried Whatman chromatography paper previously soaked into the HCN detection reagent containing copper(II) ethyl acetoacetate (5 mg) and 4,4'-methylenebis( $N, N$-dimethylaniline) (5 mg) in chloroform (1-2 ml). The $\mathrm{HCN}$ detection paper was subsequently sealed with a sticky breathable membrane (Breathe-Easy, USA Scientific) and incubated for $24 \mathrm{~h}$ at $37^{\circ} \mathrm{C}$ with shaking $(175 \mathrm{rpm})$. For quantitative values of volatile $\mathrm{HCN}$, the mean intensity of each colored spot on the membrane was measured with the ImageJ software and reported as relative units. (ii) Rhamnolipids. Detection of rhamnolipids was performed as previously described (Pinzon and Ju, 2009) with minor modifications. Briefly, $3 \mu \mathrm{l}$ of an overnight culture was spotted onto agar plates containing methylene blue and cetyl trimethylammonium bromide (CTAB) and incubated for $24 \mathrm{~h}$ at $37^{\circ} \mathrm{C}$ and subsequently at $4^{\circ} \mathrm{C}$ for $24 \mathrm{~h}$.

\section{Extraction and Purification of Rhamnolipids}

Two liters of $P$. aeruginosa PA14 were grown in LB medium at $37^{\circ} \mathrm{C}$ with constant shaking $(250 \mathrm{rpm})$ for $16-18 \mathrm{~h}$. Cell-free supernatant was extracted by liquid-liquid extraction with equal volume of EtOAc. The organic extracts were concentrated on a rotary evaporator and analyzed by thin layer chromatography (TLC) on silica gel 60 plates (Merck). The column $(2.5 \mathrm{~cm} \times$ 
$35 \mathrm{~cm}$ ) was packed with silica gel in chloroform and not allowed to dry. The sample was applied on the surface of the column and eluted with chloroform/methanol ( $/ \mathrm{v}=50: 1,20: 1$ and 10:1). Each fraction was evaporated on a rotary evaporator and analyzed by HPLC-MS/MS by using Bruker amaZon X ion trap mass spectrometer (operating in autoMS(2) positive ESI mode, scanning $\mathrm{m} / \mathrm{z}$ 100-1800) coupled with a Dionex UltiMate 3000 HPLC, with an Ascentis Express C18 column $(150 \mathrm{~mm}$ $4.6 \mathrm{~mm}, 2.7 \mathrm{~m}$, Sigma-Aldrich) and A (acetonitrile with $0.1 \%$ formic acid) and B (water with $0.1 \%$ formic acid) as the mobile phases at $1 \mathrm{ml} \mathrm{min}^{-1}$. The solvent gradient was 0-45 $\mathrm{min}, 5 \%$ $100 \%$ A. Rhamnolipids containing fractions were further purified by Sephadex LH-20 and eluted with $\mathrm{MeOH}$. Pure rhamnolipids were characterized by $1 \mathrm{D}$ and $2 \mathrm{D} \mathrm{NMR}$ as well as MS/MS analysis.

\section{Statistical Analysis}

Two-tailed unpaired $t$-test was performed on log-transformed CFU values using Prism 5.0 for Mac OS X (GraphPad Software, Inc.). Spearmans' correlations coefficient $(\rho)$ was performed on log-normalized data prior to performing the correlations.

\section{RESULTS}

\section{Hydrogen Cyanide Is Necessary, but Not Sufficient for Bcc Killing in Co-Cultures}

Smalley et al. (2015) recently showed that $P$. aeruginosa-derived $\mathrm{HCN}$ was an important toxin for killing $B$. multivorans in cocultures. Using LB medium, we genetically re-confirmed that deletion of the HCN operon $(\triangle h c n A B C)$ was sufficient to reduce the advantage of $P$. aeruginosa (strain PA14) in large shaken LB co-cultures (i.e., flasks) within $24 \mathrm{~h}$ with different B. multivorans strains as well as with other Bcc species namely $B$. cenocepacia and Burkholderia dolosa (Figure 1A and Figure S1). Genetic complementation with a functional $h c n A B C$ under an arabinose inducible promoter fully restored the ability of $\triangle h c n A B C$ to produce volatile $\mathrm{HCN}$ and to kill B. cenocepacia (strain K56-2) in LB co-cultures (Figure 1B) confirming the role of the $\mathrm{HCN}$ operon in Bcc killing. Deletion of the $h c n A B C$ operon in strain PAO1 was also impaired for killing in LB co-cultures (Figure S2). Our results along with those from Smalley et al. (2015) suggest that Pseudomonas biogenic HCN is important for killing not only B. multivorans, but also other Bcc species in LB co-cultures demonstrating the broad sensitivity of the Bcc to cyanide.

To next determine whether $P$. aeruginosa could also kill Burkholderia via the release of $\mathrm{HCN}$ in non-LB media, we co-cultured B. cenocepacia K56-2 and P. aeruginosa PA14 in undefined-rich $\mathrm{BHI}$ and the chemically defined media $\mathrm{M} 9_{\mathrm{Glc}}$, M9 ${ }_{\text {CAA }}$, and SCFM (Palmer et al., 2007). Although SCFM mimics the nutritional content of CF sputum and supports the growth of P. aeruginosa (Palmer et al., 2007) and B. cenocepacia (McKeon et al., 2011), S. aureus poorly grows in SCFM (Figure S3) demonstrating that this medium lacks components required for the growth of some common pathogens found in the CF airways. In addition to those media, we also co-cultured $B$. cenocepacia and $P$. aeruginosa in diluted $\mathrm{LB}$ and $\mathrm{BHI}\left(\mathrm{LB}_{1 / 10}, \mathrm{LB}_{1 / 4}, \mathrm{BHI}_{1 / 10}\right.$, $\left.\mathrm{BHI}_{1 / 4}\right)$. Unlike undiluted $\mathrm{LB}$, no other culture media led to
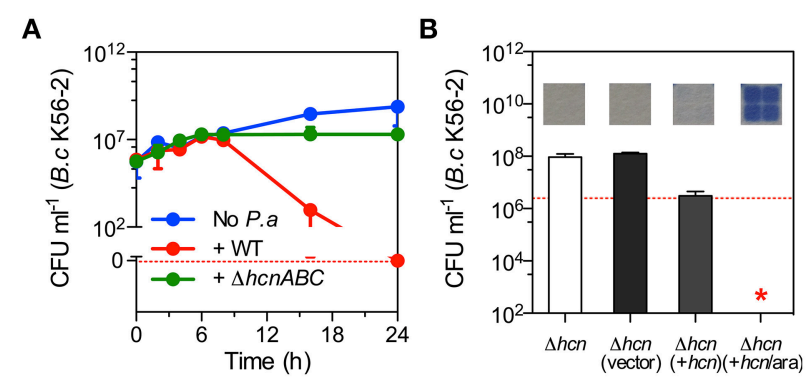

C
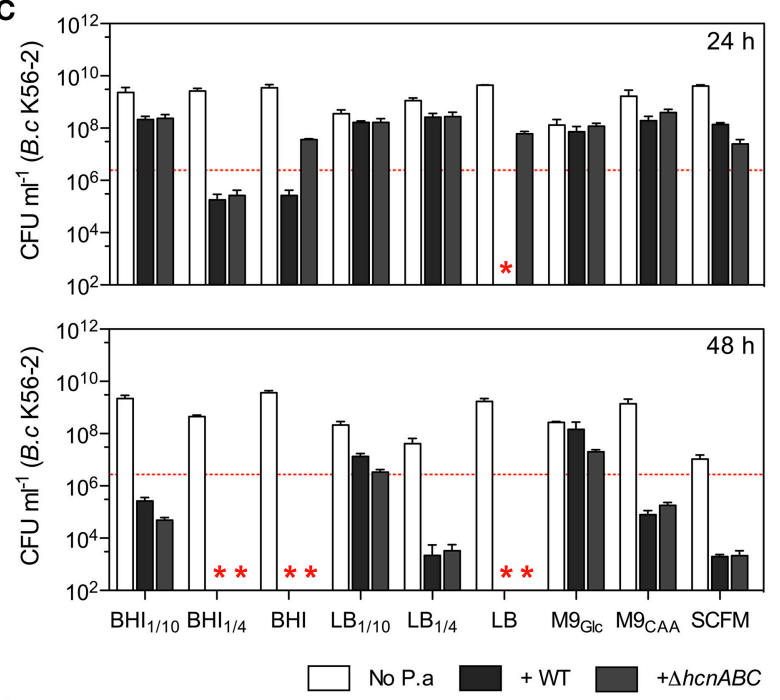

D

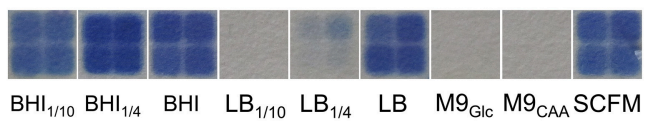

FIGURE 1 | Hydrogen cyanide-mediated killing is sensitive to growth conditions. (A) Growth of B. cenocepacia K56-2 in monoculture (blue line) and in co-culture with WT (red line) $P$. aeruginosa (strain PA14) or its $\mathrm{HCN}$-deficient mutant strain $\triangle$ hcn $A B C$ (green line) over $24 \mathrm{~h}$ in LB shaken cultures. (B) Complementation in trans of $\triangle h c n A B C(\Delta h c n)$ with pMQ72hcnABC ( $+h c n)$ when induced with $0.1 \%$ arabinose (+hcn/ara) fully restored $B$. cenocepacia K56-2 killing in LB co-cultures. (C) Release of volatile HCN by WT P. aeruginosa (strain PA14) in different culture media and the survival of B. cenocepacia (strain K56-2) in co-cultures after 24 (top) and $48 \mathrm{~h}$ (bottom) with WT P. aeruginosa (black bars) or $\triangle$ hcnABC (gray bars). (D) Detection of volatile HCN from $P$. aeruginosa PA14 cultures in different growth media. Data reported represent the mean \pm SD of a minimum of three replicates. Dotted red line in (A) represents the 0 value while it represents CFUs of B. cenocepacia K56-2 at time $0\left(\sim 2 \times 10^{6} \mathrm{CFU} \mathrm{ml}{ }^{-1}\right)$ in $\mathbf{( B , C ) . ~ R e d ~}$ stars in $\mathbf{( B , C )}$ represent the absence of $B$. cenocepacia K56-2 CFU recovered from the co-cultures or below the detection limits.

complete eradication of B. cenocepacia within $24 \mathrm{~h}$ in mixed cultures (Figure 1C). However, complete killing occurred within $48 \mathrm{~h}$ for co-cultures in $\mathrm{BHI}$ and $\mathrm{BHI}_{1 / 4}$ while a constant increase in Burkholderia killing was observed for all other tested media over a period of $48 \mathrm{~h}$ with the exception of $\mathrm{M} 9_{G l c}$ (Figure 1C). However, although the release of biogenic volatile HCN by WT $P$. aeruginosa PA14 was detected in the majority of the tested culture media with the exception of $\mathrm{LB}_{1 / 10}, \mathrm{M} 9_{G l c}$, and $\mathrm{M} 9_{C A A}$ (Figure 1D) co-cultures with $\triangle h c n A B C$ led to increased viability of B. cenocepacia K56-2 compared to the WT parent PA14 only 
in $\mathrm{LB}$ and BHI while minimal differences occurred with the other media over a period of $24 \mathrm{~h}$ (Figure 1C). Interestingly, survival of $B$. cenocepacia with $\triangle h c n A B C$ in both $\mathrm{LB}$ and $\mathrm{BHI}$ was completely lost within $48 \mathrm{~h}$ (Figure 1C) demonstrating that other toxic molecules reached inhibitory concentrations after 24 h. Accumulation of inhibitory concentrations of non-HCN toxic metabolites over time was not due to continued growth since the viable population of $P$. aeruginosa was similar between 24 and 48 h (Figure S4).

Altogether, our results demonstrate that $\mathrm{HCN}$ production is necessary, but not sufficient for complete $B$. cenocepacia killing in large shaken planktonic cultures. Although co-culture in undiluted LB medium was the only condition in which B. cenocepacia K56-2 was eradicated within 24 h (Figure 1C), our results suggest that competition between $P$. aeruginosa and $\mathrm{Bcc}$ species in liquid co-cultures is both medium- and growth phase-dependent (Figure 1 and Figure S5), for which conditions reached in rich media (i.e., $\mathrm{LB}$ and $\mathrm{BHI}$ ) seem to be favorable for effective Burkholderia killing. In addition, accumulation of $P$. aeruginosa non-HCN toxic metabolites over time is sufficient for complete Burkholderia killing in some, but not all growth media.

\section{The Susceptibility of B. cenocepacia K56-2 to Cyanide and Other $\boldsymbol{P}$. aeruginosa-Derived Toxins Is Greater in Undefined Rich Culture Media}

The lack of efficient HCN-mediated killing by $P$. aeruginosa PA14 in chemically defined or diluted rich media within $24 \mathrm{~h}$ (Figure 1C) compared to rich LB medium could be the result of lower toxin concentrations (i.e., sub-inhibitory) and/or increased Burkholderia tolerance to cyanide and other toxic molecules in these particular conditions. To address the latter, we first determined the minimal inhibitory concentration (MIC) of potassium cyanide $(\mathrm{KCN})$, as a source of cyanide, in culture media for which volatile $\mathrm{HCN}$ was produced and detected.

Using 96-well microtiter plates, we first showed that the susceptibility of B. cenocepacia K56-2 to KCN was greatly affected by culture medium with MICs spanning from 0.4 to $>25 \mathrm{mM}$. Assays done in undiluted rich media (i.e., BHI and LB) gave the lowest MICs and those done in synthetic media (SCFM) gave the highest MICs (Table 1). The high cyanide susceptibility in LB medium was further demonstrated within the Bcc by testing four B. multivorans strains (C1576, C5393, CF-A1-1, and ATCC 17616) and one B. dolosa strain (PC543) with MIC values for KCN between 1.6 and $3.2 \mathrm{mM}$ in comparison to 12.5 and $>25 \mathrm{mM}$ for $P$. aeruginosa PA14 and $S$. aureus (strain RN6390), respectively (Table 2). Growth profiles of $B$. cenocepacia K56-2 were different in these culture media, which may explain some of the differences in susceptibility to cyanide. In fact, the lag phase was longer in chemically defined media compared to rich media like LB and BHI (Figure S6).

Although the susceptibility of $B$. cenocepacia K56-2 to cyanide was highly dependent on culture media (Figure 1C and Table 1), the bioactivity of other $P$. aeruginosa toxins (Figure S1 and Tomlin et al., 2001; Bakkal et al., 2010; Costello
TABLE 1 | Susceptibility of $B$. cenocepacia K56-2 to $P$. aeruginosa-derived small molecules.

\begin{tabular}{|c|c|c|c|c|c|}
\hline Medium & $\begin{array}{c}\text { Spent } \\
\text { medium (\%)a }\end{array}$ & $\begin{array}{l}\mathrm{KCN} \\
(\mathrm{mM})^{\mathrm{b}}\end{array}$ & $\begin{array}{l}\text { PYO }(\mu \mathrm{g} \\
\left.\mathrm{ml}^{-1}\right)^{\mathrm{b}}\end{array}$ & $\begin{array}{l}\text { PCA }(\mu \mathrm{g} \\
\left.\mathrm{ml}^{-1}\right)^{\mathrm{b}}\end{array}$ & $\operatorname{RLs}\left(\mu \mathrm{g} \mathrm{ml}^{-1}\right)^{\mathrm{b}}$ \\
\hline LB & 50 & 0.8 & $>300$ & $>60$ & $\geq 600$ \\
\hline $\mathrm{LB}_{1 / 4}$ & $>50$ & 1.6 & $>300$ & $>60$ & N.D. \\
\hline $\mathrm{LB}_{1 / 10}$ & 50 & 3.2 & $>300$ & $>60$ & N.D. \\
\hline $\mathrm{BHI}$ & $>50$ & 0.4 & $>300$ & 7.5 & N.D. \\
\hline $\mathrm{BH}_{1 / 4}$ & $>50$ & 1.6 & $>300$ & $>60$ & N.D. \\
\hline $\mathrm{BH}_{1 / 10}$ & 12.5 & 1.6 & $>300$ & $>60$ & N.D. \\
\hline $\mathrm{M}_{\mathrm{Glc}}$ & 12.5 & N.D. & N.D. & N.D. & N.D. \\
\hline $\mathrm{M9}_{\mathrm{CAA}}$ & 12.5 & N.D. & N.D. & N.D. & N.D. \\
\hline SCFM & 25 & $>25$ & $>300$ & $>60$ & N.D. \\
\hline
\end{tabular}

MIC values after $24 \mathrm{~h}$ of growth in 96-well format using B. cenocepacia K56-2 are reported.

a Spent medium was extracted from 24-h planktonic cultures of WT P. aeruginosa PA14 grown as described in the Materials and Methods section. The antimicrobial activity of each spent medium was determined in the same growth medium for which supernatants were extracted.

${ }^{b}$ The MIC of purified compounds were determined in $\angle B$ medium.

N.D., Not determined; KCN, potassium cyanide; PYO, pyocyanin; PCA, phenazine-1carboxylic acid; RLs, di-rhamnolipids.

TABLE 2 | Cyanide susceptibility of CF associated bacterial pathogens.

\begin{tabular}{lcc}
\hline Species & Strain & KCN (mM) \\
\hline B. multivorans & C1576 & 1.6 \\
& C5393 & 3.2 \\
& CF-A1-1 & 1.6 \\
B. cenocepacia & ATCC 17616 & 1.6 \\
B. dolosa & K56-2 & $0.4-0.8$ \\
P. aeruginosa & PC543 & 3.2 \\
S. maltophilia & PA14 & 12.5 \\
S. aureus & K279a & 0.8 \\
\end{tabular}

MIC values after $24 h$ of growth are reported. Bacteria were grown in LB medium using 96-well microtiter plates and incubated aerobically at $37^{\circ} \mathrm{C}$ with constant shaking (175 rpm).

et al., 2014; Smalley et al., 2015) could also be affected which may further explain the lack of killing in cyanide-positive conditions (Figures 1C,D). To address this possibility, we extracted supernatants from 24-h monocultures of $P$. aeruginosa PA14 grown in all nine culture media (i.e., $\mathrm{LB}, \mathrm{LB}_{1 / 4}, \mathrm{LB}_{1 / 10}$, $\mathrm{BHI}, \mathrm{BHI}_{1 / 4}, \mathrm{BHI}_{1 / 10}, \mathrm{M}_{\mathrm{Glc}}, \mathrm{M} 9_{\mathrm{CAA}}$, and SCFM) previously tested in co-cultures (Figure $\mathbf{1 C}$ ) to determine their respective antibiotic-like activity. All tested $P$. aeruginosa spent media had different levels of toxicity on the growth of $B$. cenocepacia K56-2 with MIC values spanning from 12.5 to greater than $50 \%$ where supernatants from the chemically defined M9 and SCFM being the most toxic (Table 1). In addition, the MIC of spent medium from $\triangle h c n A B C$ LB cultures was similar $(\sim 25-50 \%)$ to its WT parent demonstrating that non-HCN toxins are active against Burkholderia in co-cultures (Figure 1C and Figure S1). Furthermore, since $\mathrm{HCN}$ is highly volatile, its concentration in spent medium after extraction is likely sub-inhibitory. These results suggested that the toxicity of 
Pseudomonas was maintained in most culture media; including those from chemically defined M9 or SCFM that were not highly toxic in co-cultures within $24 \mathrm{~h}$ (Figure 1C).

The increased susceptibility of B. cenocepacia to $P$. aeruginosa and cyanide in rich medium may suggest that the susceptibility of Burkholderia may vary in function of the growth medium. To demonstrate this possibility, we determined the MIC of Pseudomonas spent medium from SCFM cultures (low MIC; Table 1) in LB medium as well as Pseudomonas spent medium from LB cultures in SCFM medium. Interestingly, the MIC of SCFM spent medium required to inhibit the growth of B. cenocepacia K56-2 went from $25 \%$ in SCFM to less than $6.25 \%$ in LB medium while the MIC of LB spent medium stayed at $50 \%$ in both culture media. These results demonstrated that higher amounts of Pseudomonas toxins were present in SCFM compared to $\mathrm{LB}$ cultures since the MIC of those in LB medium was much lower than in SCFM confirming that $B$. cenocepacia is more susceptible to $P$. aeruginosa toxins in rich medium like LB than SCFM. Other toxins like phenazines and rhamnolipids were shown using mutant strains to have antibiotic-like activity in LB co-cultures against $B$. multivorans (Smalley et al., 2015), which we re-confirmed in other Bcc species (Figure S1). However, purified pyocyanin (PYO) and phenazine-1-carboxylic acid (PCA), two phenazines produced by $P$. aeruginosa (Price-Whelan et al., 2006), did not exhibit much toxicity in all tested growth conditions with the exception of PCA in BHI (Table 1). In addition, purified di-rhamnolipids (RLs) did not have any antimicrobial activity against B. cenocepacia K562 in LB medium (Table 1). These results demonstrated that the toxicity of other potential small molecules could also be impacted by growth medium.

Overall, our results demonstrate that Pseudomonas toxic metabolites are present at inhibitory concentrations when grown in chemically defined media like SCFM, but the susceptibility of B. cenocepacia K56-2 to these chemicals, including cyanide, is greatly influenced by environmental factors (i.e., culture medium) for which $B$. cenocepacia is more sensitive in rich medium. Altogether, it demonstrates that the growing environment plays a critical role in the ability of B. cenocepacia to tolerate the toxicity of $P$. aeruginosa mediated by the release of small molecules such as cyanide and phenazines.

\section{The Relationship between $P$. aeruginosa Quorum Sensing, Hydrogen Cyanide Production and Burkholderia Killing}

Loss-of-function mutations in genes involved in quorum sensing (QS) regulation are often isolated in host-adapted $P$. aeruginosa CF isolates (Smith et al., 2006; Hoffman et al., 2009; Cullen and McClean, 2015). P. aeruginosa uses three QS systems (Las, Rhl, and PQS) as global regulators to coordinate the regulation of target gene networks, including the $h c n A B C$ operon (Jimenez et al., 2012; Tashiro et al., 2013) and mutants in the Las or the Rhl QS system were shown to be less toxic for B. multivorans in LB co-cultures (Smalley et al., 2015) while PQS negative supernatants had no growth inhibition on $B$. cenocepacia (Costello et al., 2014). Here, we wanted to determine the contribution of each of the three QS systems with respect to Burkholderia killing and $\mathrm{HCN}$ production.

Where optimal killing occurs, in LB co-cultures, all tested Bcc strains representing $B$. multivorans, $B$. cenocepacia, and $B$. dolosa showed increased survival within $24 \mathrm{~h}$ in the presence of any of the three QS-deficient mutants $(\triangle l a s R, r h l R$, and $\triangle p q s A$ ) compared to their wild-type (WT) parent (Figure 2A and Figure S7). Introduction of the $\triangle h c n A B C$ mutation into $\triangle l a s R$ and $\triangle p q s A$ mutant strains significantly increased the viability of $B$. cenocepacia K56-2 while no difference was observed when the same mutation was added into $r h l R$ mutant strain (Figure 2A). In agreement with these results, both $\triangle$ las $R$ and $\triangle p q s A$ still produced $H C N$ unlike $r h l R$ for which the levels of detected volatile $\mathrm{HCN}$ were severely reduced compared to WT (Figure 2B). To next determine whether survival of $B$. cenocepacia $\mathrm{K} 56-2$ in co-cultures with $\triangle p q s A$ was mediated via PQS or 2-heptyl-4-quinolone (HHQ), we tested a $\Delta p q s H$ mutant strain. HHQ, the precursor of PQS, is produced by the pqsABCDE operon, while PqsH further converts HHQ into PQS (Déziel et al., 2004; Tashiro et al., 2013). Unlike $\Delta p q s A$ (i.e., HHQ negative, PQS negative), $\Delta p q s H$ (i.e., HHQ positive, PQS negative) was still fully toxic in co-cultures while $\Delta p q s R$ (i.e., HHQ negative, $\mathrm{PQS}$ negative), the positive regulator of the operon was not (Figure S8). HHQ and PQS can bind the PqsR regulator to coordinate the expression of target genes (Jimenez

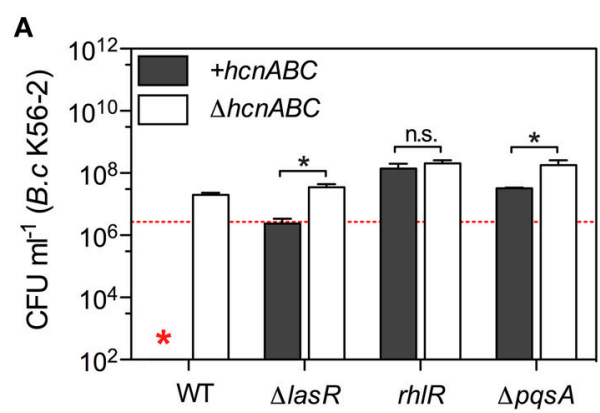

B

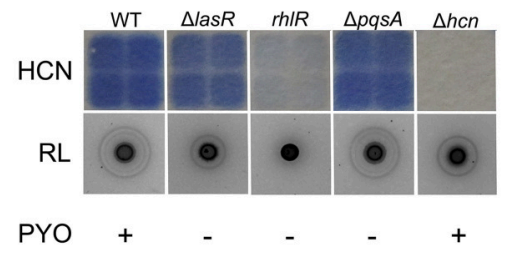

FIGURE 2 | Relationships between quorum sensing, $\mathrm{HCN}$ production, and Burkholderia killing. (A) Viability of B. cenocepacia K56-2 after $24 \mathrm{~h}$ in mixed cultures WT P. aeruginosa PA14 and its QS-deficient mutant strains $\Delta$ las $R, r h / R$, and $\triangle p q s A$ positive (gray bars; $+h c n A B C$ ) or negative (white bars; $\triangle h c n A B C$ ) for the $h c n A B C$ operon. (B) Phenotypic profiling of $P$. aeruginosa $\mathrm{PA}$ 14-derived strains tested in (A) for their in vitro ability to produce volatile HCN, rhamnolipids (RL), and pyocyanin (PYO). Red star represents the absence of B. cenocepacia K56-2 CFU recovered from the co-cultures or below the detection limits. Data reported represent the mean $\pm \mathrm{SD}$ of a minimum of three replicates. Dotted red line in $\mathbf{( A )}$ represents $B$. cenocepacia K56-2 CFUs at time $0\left(\sim 2 \times 10^{6} \mathrm{CFU} \mathrm{ml}{ }^{-1}\right)$. Asterisks indicate values significantly different by two-tailed unpaired $t$-test: ${ }^{*} P \leq 0.001$ and n.s. (not significant). 
et al., 2012), consequently our genetic results can only confirm that mechanism(s) of Burkholderia killing regulated by the PQS system are PqsR-dependent since we did we did not test a mutant strain that was PQS positive and HHQ negative to separate the two specific pathways.

Interestingly, disruption of $r h l R$ allowed all tested Bcc strains to reach cell densities close to those normally achieved during monoculture growth, which was not always the case for cocultures with $\triangle$ lasR or $\triangle p q s A$ mutant strains (Figure $2 \mathbf{A}$ and Figure S7). In all cases, the increased survival of Burkholderia was not due to the reduced ability of the QS-deficient mutants to compete in mixed cultures, but rather their reduced toxicity toward Bcc species, since viable counts of the $P$. aeruginosa strains were equivalent to those of the WT parent and comparable to monoculture growth (Figure S9). These results demonstrate that while $P$. aeruginosa-derived $\mathrm{HCN}$ is a major toxin for the $\mathrm{Bcc}$, additional factors are required for effective killing that are LasR-, RhlR-, and PqsR-regulated such as phenazines, rhamnolipids (Figure 2B and Figure S1; Smalley et al., 2015), pyoverdine via iron chelation (Costello et al., 2014; Tyrrell et al., 2015), and other molecules yet to be identified.

\section{The Burkholderia Killing Trait Is Diverse among Host-Adapted $P$, aeruginosa CF Isolates}

Pathogenic bacteria adapt and diversify phenotypically in polymicrobial infections of the lower airways (Cullen and McClean, 2015). P. aeruginosa has been shown to acquire numerous adaptive mutations in the airways (Smith et al., 2006) and to establish phenotypically diverse populations (Mowat et al., 2011; Workentine et al., 2013; Jorth et al., 2015). To assess whether the long-term adaptation to the CF lung may impact the toxicity of $P$. aeruginosa, we evaluated a collection of Pseudomonas $\mathrm{CF}$ isolates belonging to the Prairie epidemic strain (PES) (Parkins et al., 2014). We tested 26 representative clinical isolates that were obtained from a single patient over a period of 10 months during both exacerbation and clinically stable periods (Figure 3B) and for which phenotypic diversity (i.e., colony morphotypes, growth, motility, QS signals, siderophores, proteolytic activity, and antibiotic resistance) was previously shown (Workentine et al., 2013).

Using LB medium as optimal Burkholderia killing growth medium (Figure 1C), we co-cultured 26 P. aeruginosa CF isolates in pairs with $B$. cenocepacia K56-2 and found that only one isolate $(\# 121)$ was able to eradicate $B$. cenocepacia from the mixed culture while others had intermediate to no toxicity (Figure 3A). Interestingly, half of the co-culture pairs were dominated by $B$. cenocepacia rather than $P$. aeruginosa after $24 \mathrm{~h}$ (Figures 3A,B; green circles) suggesting either neutral or positive interactions with these strains. All tested $26 \mathrm{P}$. aeruginosa CF isolates were capable of producing volatile $\mathrm{HCN}$ in vitro at similar or higher levels than those from the laboratory strains PA14 and PAO1 (Figure 3B). Statistical correlation analysis comparing B. cenocepacia K56-2 CFUs after $24 \mathrm{~h}$ in each co-culture pair was directly compared to previously evaluated phenotypes of the respective 26 P. aeruginosa PES isolates (Workentine et al.,
2013) to find possible predictors of Burkholderia survival or killing. Spearman rank correlation did not show any statistical correlation of B. cenocepacia CFUs with HCN, QS signals (C4and C12-HSL), or growth in LB (Figure S10).

Using a small subset of evolved $P$. aeruginosa $\mathrm{CF}$ isolates from a single patient, we demonstrated using simple pairwise co-cultures the full spectrum of competitive interactions that could possibly occur in complex in vivo communities where both species can dominate each other or establish population equilibrium. Further, HCN production alone did not explain Burkholderia killing in co-cultures with clinical isolates, however additional factors may be required for effective killing reinforcing the concept of multifactorial strategy.

\section{The Composition of Bacterial Communities Influences the Tolerance of $B$. cenocepacia to Cyanide}

Chronic infections affecting the lower airways are polymicrobial (Huang and Lynch, 2011; Han et al., 2012; Dickson et al., 2014; Surette, 2014), consequently we next evaluated whether the presence of other CF pathogens namely $S$. aureus and $S$. maltophilia would impact the tolerance of $B$. cenocepacia K56-2 to cyanide in LB co-cultures. The tolerance of $S$. maltophilia (strain K279a) to cyanide (i.e., $\mathrm{KCN}$ ) in $\mathrm{LB}$ was comparable to Bcc species while S. aureus (strain RN6390) was fully resistant (Table 2). The cyanide resistance profiles of these two pathogens span the concentration spectrum making these attractive candidates to use to determine their impact on Burkholderia cyanide tolerance in mixed communities.

We monitored the viability of $B$. cenocepacia $\mathrm{K} 56-2$ in the presence of increasing concentrations of KCN $(0-1000 \mu \mathrm{M})$ in monocultures and co-cultures with $S$. aureus RN6390, S. maltophilia K279a, and the less toxic rhlR mutant of $P$. aeruginosa strain PA14 after $24 \mathrm{~h}$ in LB medium. In monocultures, the growth/viability of $B$. cenocepacia K56-2 was severely impaired with $500 \mu \mathrm{M} \mathrm{KCN}$ while it was fully eradicated with $1000 \mu \mathrm{M}$ (Figure 4). The addition of $S$. maltophilia increased the growth of B. cenocepacia K56-2 with $500 \mu \mathrm{M} \mathrm{KCN} \mathrm{(Figure} \mathrm{4C)} \mathrm{while} \mathrm{the}$ addition of $S$. aureus or rhlR allowed Burkholderia to survive and grow with KCN concentrations up to $1000 \mu \mathrm{M}$ (Figures 4A,B). At the species level, the cyanide tolerance observed in co-cultures reflected what was previously determined in monocultures with $S$. aureus being the most tolerant followed by $P$. aeruginosa, S. maltophilia, and B. cenocepacia (Table 2 and Figure 4). In agreement with the latter, the viability of $B$. cenocepacia in co-cultures with inhibitory concentrations of $\mathrm{KCN}$ followed a similar trend with $S$. aureus being the most protective against cyanide toxicity (Figure 4).

These results suggested that molecule(s) released in the extracellular milieu by other bacterial species could allow Burkholderia to tolerate higher concentrations of KCN. To test this hypothesis and the potential antagonistic effects between bacterial spent medium and $\mathrm{KCN}$, we used the fractional inhibitory concentration index (FICI) in checkerboard liquid cultures as a measure of the interaction between two antimicrobial agents (Odds, 2003; Pillai et al., 2005). FICI 

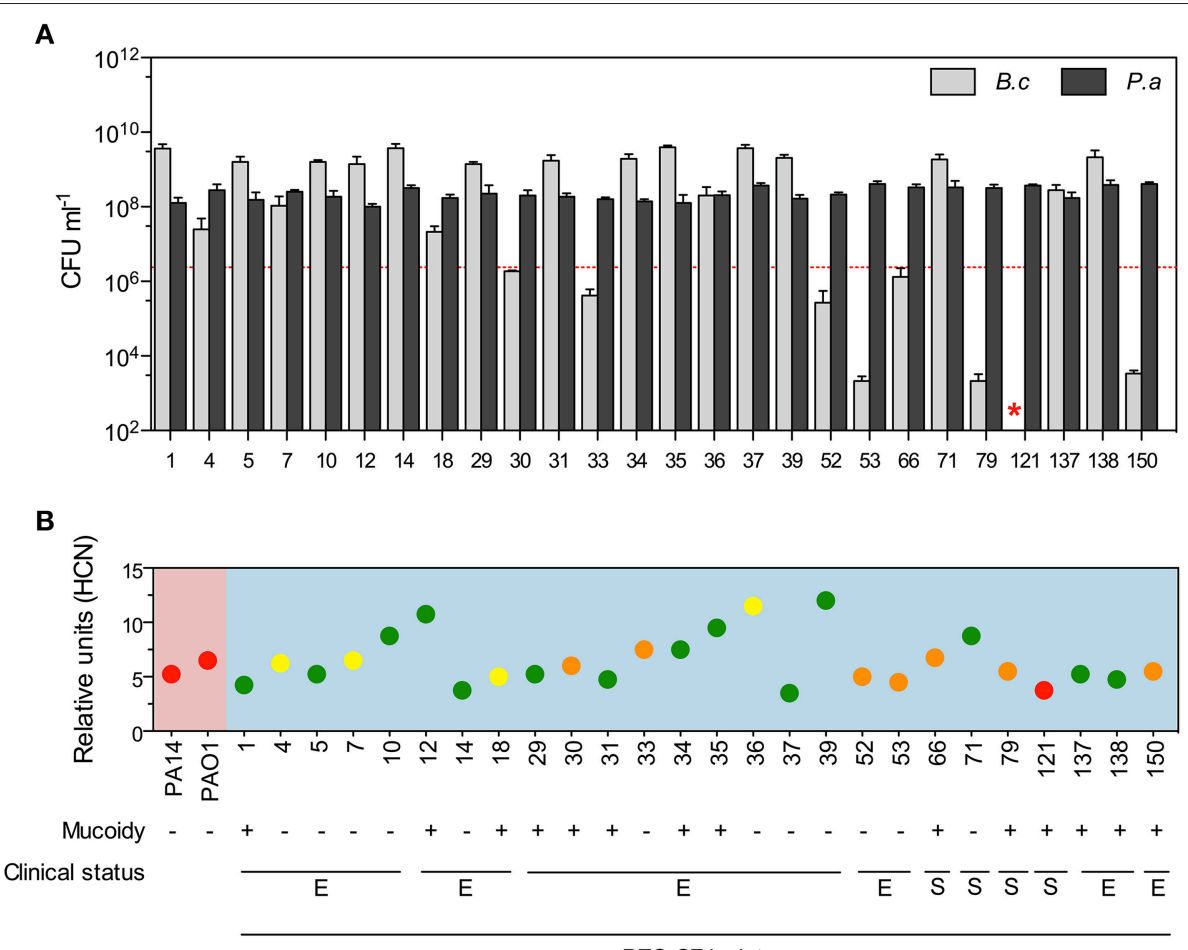

PES CF isolates

FIGURE 3 | Burkholderia killing diversity among P. aeruginosa CF isolates. (A) B. cenocepacia K56-2 was co-cultured in LB medium in pairs with 26 P. aeruginosa CF isolates collected from a single patient during different exacerbation (E) and clinically stable (S) periods where survival/growth of each species was monitored after 24 h. (B) Relative levels of volatile HCN produced by each PES isolates (blue background) compared to the laboratory strain PA14 and PAO1 (pink background) within $24 \mathrm{~h}$ in LB cultures and their ability to kill or not kill B. cenocepacia K56-2 in co-cultures are represented by the dots in green, yellow, orange, and red. The meaning of the colors are as follow: total eradication of Burkholderia (red), growth/survival of Burkholderia above (yellow) or below (orange) the initial inoculum (dotted red line in A), and higher numbers of Burkholderia compared to P. aeruginosa (green). Red star represents the absence of B. cenocepacia K56-2 CFUs recovered from the co-cultures or below the detection limits. Data reported in (A) represent the mean \pm SD of a minimum of three replicates and the dotted red line represents $B$. cenocepacia K56-2 CFUs at time 0. Values for volatile HCN quantification (relative units) in (B) represent the mean \pm SD of four replicates.

of $\leq 0.5,>4.0$, and $>0.5-4.0$ indicate synergy, antagonism, and no interaction, respectively (Odds, 2003). Addition of spent medium from $P$. aeruginosa PA14 or S. aureus RN6390 increased the tolerance of $B$. cenocepacia K56-2 to cyanide with FICI of 4.0625 and $>8.125$, respectively (Figure 5A), confirming the antagonistic interactions between supernatants from these two bacterial species and KCN. Spent medium from either S. maltophilia K279a or B. cenocepacia K56-2 in combination with KCN resulted in an FICI of 2.0625 and 0.75 , respectively representing no detectable synergistic or antagonistic interaction. These results corroborated the co-culture observations (Figure 4) for which both $P$. aeruginosa PA14 and S. aureus RN6390 increased the cyanide tolerance of B. cenocepacia K56-2 in mixed communities with S. aureus being the most protective in both cases (Figures 4,5A). Heat treatment of $P$. aeruginosa PA14 and S. aureus RN6390 supernatants abolished the antagonistic interactions with FICI of 0.75 and 1.0, respectively (Figure 5), suggesting that heat labile extracellular molecule(s) from these bacterial species could be protective against cyanide. Altogether these results demonstrate that the overall composition of the microbial community can have profound effects on competitive mechanisms mediated by the exchange of toxic metabolites like cyanide.

\section{DISCUSSION}

Microbial interactions (e.g., positive and competitive) in polymicrobial infections can have profound effects on disease progression (Peters et al., 2012; Murray et al., 2014; Short et al., 2014) and antibiotic resistance (Bernier and Surette, 2013). Although, the concept of cooperation and polymicrobial synergy is well documented (Peters et al., 2012; Murray et al., 2014; Short et al., 2014) not much is known about the role of competition in mixed bacterial infections. Co-infections or succession of pathogens in the CF airways could involve interspecies competition and consequently change the composition of the community with the potential to affect the progression of the infection. As example, $P$. aeruginosa uses a multifactorial strategy to outcompete $S$. aureus in co-cultures (Filkins et al., 2015), which could explain, in certain cases, the replacement of $S$. aureus in CF airways at late adolescence and adulthood (Harrison, 2007).

In the current study, we focused on expanding our in vitro understanding of competitive interactions mainly mediated by cyanide that was recently proposed to be an important strategy used by $P$. aeruginosa to kill $B$. multivorans in LB co-cultures (Smalley et al., 2015). HCN is a poison produced by $P$. aeruginosa in the CF lung that can affect host immune functions (Anderson 


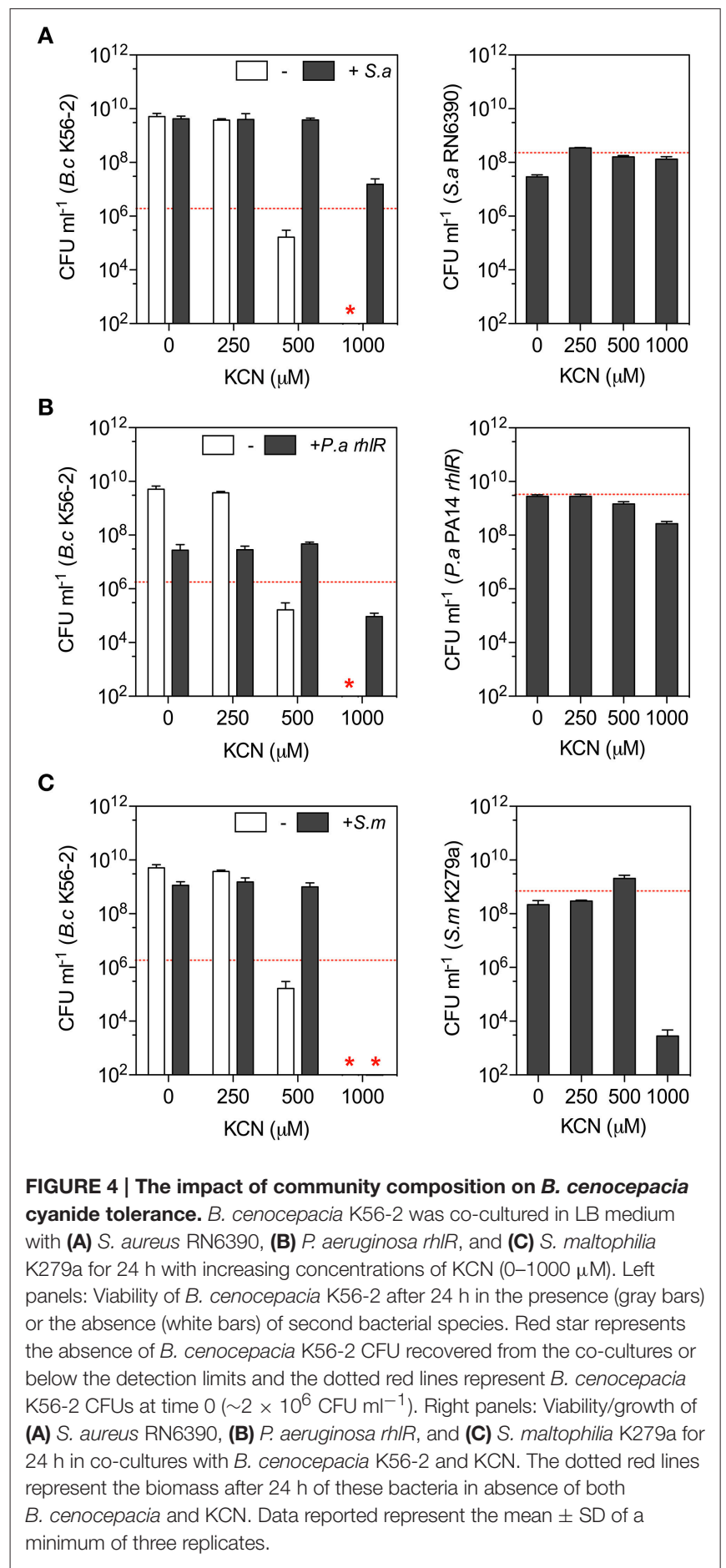

et al., 2010; Lenney and Gilchrist, 2011; Nair et al., 2014), with also the potential to outcompete other bacterial species in polymicrobial infections and consequently reduce species diversity of these complex communities. However, we showed that the competitive efficacy of $P$. aeruginosa toward the Bcc via the release of toxins, including cyanide, depends on several factors such as the growth medium, the in vivo adaptive evolution of $P$. aeruginosa, and the presence of other bacteria in the mixed community.

Deletion of the $h c n A B C$ operon increased the viability of all tested Bcc species in LB co-cultures with $P$. aeruginosa within $24 \mathrm{~h}$ (Figure 1 and Figure S1), however loss of HCN failed to maintain the viability of $B$. cenocepacia over $48 \mathrm{~h}$ in rich LB and BHI media (Figure 1C) suggesting that accumulation of non-HCN toxins reached inhibitory concentrations. These results are in agreement with the fact that Bcc killing is QSmediated (Figure 2 and Figure S7; Costello et al., 2014; Smalley et al., 2015), which also involve the QS-regulated phenazines and rhamnolipids (Figure S1). Interestingly, B. cenocepacia was more susceptible to cyanide and other toxins (e.g., spent medium) in rich medium than in SCFM (Table 1). Although differences in nutrients between culture media may affect metabolism and consequently tolerance to $P$. aeruginosa toxins, we showed that the growth profile of $B$. cenocepacia was different with a longer lag phase in SCFM compared to LB and BHI media (Figure S6). An extended lag phase could impact the tolerance of Burkholderia to $P$. aeruginosa toxins, similarly to the increased tolerance of evolved E. coli populations due to longer lag phase (Fridman et al., 2014). Whether SCFM reflects the nutritional content of the CF lung is an important question, but knowing that growth of S. aureus is not supported in SCFM (Figure S3) suggests that key components are missing to support the growth of CF pathogens other than P. aeruginosa and the Bcc.

Biogenic $\mathrm{HCN}$ has pleiotropic biological roles since it can act as a classical virulence factor (Anderson et al., 2010; Zdor, 2015), a biocontrol agent of plant fungal diseases (Blumer and Haas, 2000; Zdor, 2015), a policing agent controlling the emergence of social cheaters (Wang et al., 2015), and results from our study suggest that it could be used as a strategy to outcompete other bacterial species in polymicrobial infections and consequently reduce species diversity of these complex communities. How the $\mathrm{Bcc}$ is killed by cyanide remains an unanswered question, but cyanide generally poisons susceptible eukaryotic and prokaryotic cells by interfering with the aerobic respiratory chain by inhibiting cytochrome c oxidase (Lenney and Gilchrist, 2011; Wybouw et al., 2014). To tolerate higher concentrations of cyanide, $P$. aeruginosa possesses cyanideresistant oxidases and the rhodanese RhdA that converts cyanide into the less toxic thiocyanate (Cunningham and Williams, 1995; Cooper et al., 2003; Cipollone et al., 2007a,b). Results from our study and Smalley et al. (2015) conclusively demonstrate the high sensitivity of the Bcc to cyanide, but this is rather surprising since it was previously suggested that the Bcc produced $\mathrm{HCN}$ (Ryall et al., 2008; Neerincx et al., 2015). However, another study failed to detect volatile HCN from Bcc grown as biofilms or in liquid or from breath samples of Burkholderia-infected CF patients (Gilchrist et al., 2013). Although these studies are controversial, we did not detect any volatile $\mathrm{HCN}$ released from 41 strains representing nine Bcc species within $24 \mathrm{~h}$ in LB cultures (data not shown). Although 3 potential hcn $A B C$ homologs were found in B. cenocepacia (Ryall et al., 2008) and the discrepancy between different studies, further work is required to better understand the possible relationship between cyanide production and sensitivity in the Bcc. 
A
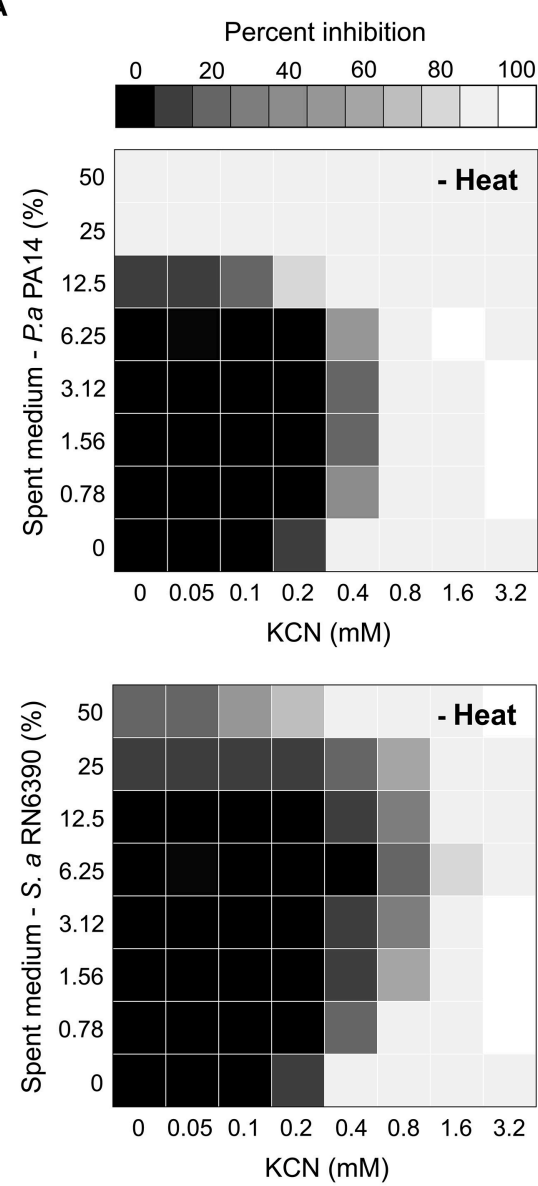

B
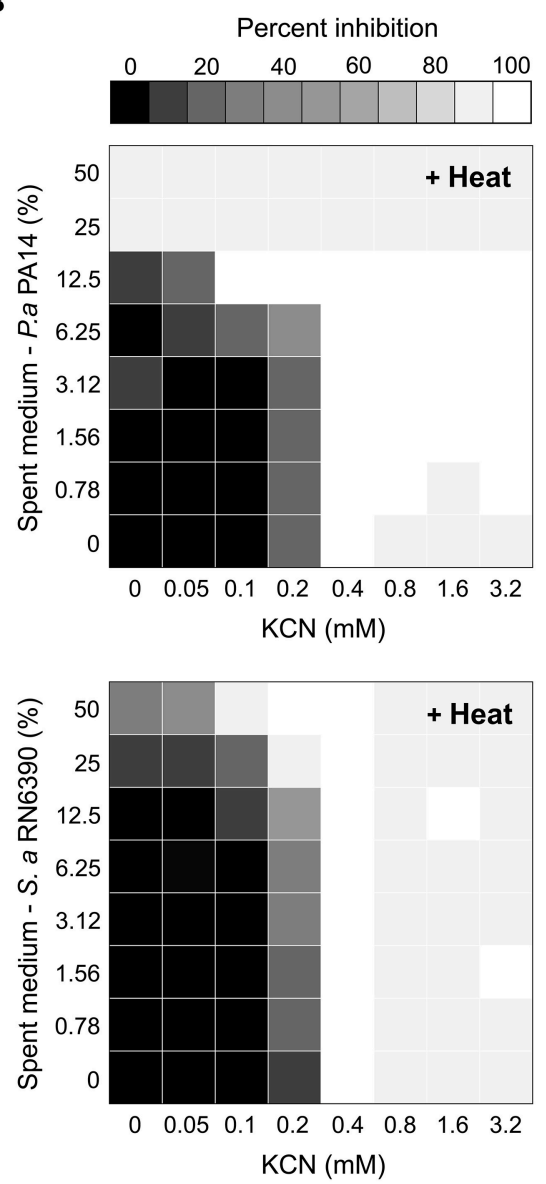

FIGURE 5 | The antagonistic effect of $P$ aeruginosa and $S$. aureus supernatants on the antimicrobial activity of potassium cyanide against $B$. cenocepacia. Heat plots showing the antagonistic growth effect of $P$. aeruginosa PA14 (top) and S. aureus RN6390 (bottom) spent media on the toxicity of KCN on the growth of B. cenocepacia K56-2 before (A) and after (B) heat treatments. Antagonism mediated by heat labile molecule(s) was evident as the MIC for KCN increased with untreated spent medium while being unaffected after heat treatments.

As the competitive interactions described here occur mainly through metabolite production it is important to consider the capacity of an entire population rather than individual isolates. Knowing that populations of $P$. aeruginosa are phenotypically and genetically heterogeneous in the CF lung (Smith et al., 2006; Mowat et al., 2011; Workentine et al., 2013) and that adaptive mutations may impact the production of toxins, testing a large number of isolates is likely the only valid in vitro approach to demonstrate an overall lack of killing at the population level. Regional diversification of $P$. aeruginosa occurs over time in the CF lung (Jorth et al., 2015) which could potentially lead to areas where active mixed infections with Burkholderia would be possible while in other areas of the lung this may not happen due to $\mathrm{HCN}$-independent and -dependent competition. However, due to the nature of sputum collection, it is for now impossible to determine whether the isolates come from one or several regions of the lungs. Using 26 P. aeruginosa clinical isolates collected over a period of 10 months from a single patient, we demonstrated that long-term adaptation of Pseudomonas to the CF lung had profound effects on the viability of Burkholderia in optimal killing LB co-cultures (Figure 3). In fact, B. cenocepacia became the dominant organism within the community with half of the tested CF host-adapted $P$. aeruginosa isolates, demonstrating that competitive genetic factors may be under selection in host environments. These results may explain, in part, why Bcc species dominate the $P$. aeruginosa population in certain co-infected CF patients (Schwab et al., 2014; Carmody et al., 2015), which may coincide with a reduced overall Pseudomonas toxicity at the population level. Loss-of-function mutations in QS genes are common in host-adapted $P$. aeruginosa (Smith et al., 2006; Hoffman et al., 2009; Cullen and McClean, 2015) and the reduced toxicity of $\triangle l a s R, r h l R$, and $\triangle p q s A$ in co-cultures (Figure $2 \mathrm{~A}$ and Figure S7) precisely re-confirm their role in interspecies competition (Park et al., 2005; Hoffman et al., 2006; Filkins et al., 2015; Smalley et al., 2015). In addition, host adapted isolates may have additional competitive mechanism(s) not identified in this study since one isolate was fully toxic in co-culture but produced relatively low levels of HCN (Figure 3). 
There is a possible association between siderophores and Burkholderia killing since pyoverdine was shown to have antibiotic activity against B. cenocepacia (Costello et al., 2014). However, Tyrrell et al. (2015) elegantly demonstrated that growth inhibition of B. cenocepacia by pyoverdine was likely related to its iron chelating ability rather than direct killing as previously proposed (Costello et al., 2014), which would be in agreement with our co-culture data with the mutants $p v d S$ and $p v d L$ (Figure S11). Pyoverdine is regulated by the PQS system (Costello et al., 2014), but the lack of $B$. cenocepacia survival with $p v d S$ and $p v d L$ mutants cannot explain that $\triangle p q s A$ and $\triangle p q s R$ were less toxic in co-cultures although still positive for $\mathrm{HCN}$ (Figure 2). Other than phenazines (Figure 2 and Figure S1), the reduced killing phenotype of $\triangle p q s A$ and $\Delta p q s R$ remains unknown since purified PQS and HHQ have no antibiotic activity against $B$. cenocepacia (Costello et al., 2014). Although pyoverdine does not seem to act as an antibiotic on B. cenocepacia, it would be interesting to determine the role of iron-mediated competition in the establishment of long-term complex communities in vitro. Investigating siderophore-mediated interactions would be highly relevant since siderophores are important virulence factors of both P. aeruginosa and Bcc during infections. Further, pyoverdine induces expression of genes coding for the Bcc siderophore ornibactin (Tyrrell et al., 2015), while ornibactin induces gene expression in $P$. aeruginosa (Weaver and Kolter, 2004) demonstrating that iron competition has the potential to shape communities and impact pathogenesis.

In polymicrobial communities, other microorganisms may have a direct impact on competition between two species and consequently neutralize what would typically be observed in simple in vitro two-species systems. Since $P$. aeruginosa uses multiple toxins to kill $B$. cenocepacia, we had to reduce the number of negative interactions to one in order to adequately evaluate the effect of a third partner. Consequently, we replaced $P$. aeruginosa by cyanide (KCN) and evaluated the impact of nontoxic cyanide-resistant bacteria on the viability of B. cenocepacia. The addition of non-toxic cyanide-resistant bacterial species like $P$. aeruginosa rhlR or $S$. aureus increased the tolerance of $B$. cenocepacia K56-2 to cyanide in LB co-cultures (Figures 4 A,B) while less tolerant bacteria like $S$. maltophilia had a lesser impact on cyanide tolerance (Figure 4C). Interestingly, the increased cyanide tolerance conferred by $P$. aeruginosa or $S$. aureus was the result of extracellular heat labile molecule(s) that act antagonistically with cyanide (Figure 5) and consequently allowing $B$. cenocepacia to grow in the presence of cyanide concentrations typically toxic in monoculture settings (Figures 4, 5). Studies are currently underway to identify the sought after molecule(s) conferring cyanide tolerance from both $S$. aureus and $P$. aeruginosa. Neutralization of antibiotics by a third species can attenuate the inhibitory interactions between two species allowing ecological stability in well-mixed environments (Kelsic et al., 2015) while typical rock-paper-scissors interactions support microbial diversity in spatial and local environments, but not in mixed environments (Kerr et al., 2002). Our results with cyanide-resistant bacteria inhibiting negative interactions between $B$. cenocepacia and cyanide support a model where neutralization of a toxin in communities can maintain microbial diversity in well-mixed environments.

In conclusion, polymicrobial communities can involve a large number of positive and negative interactions. Here, we show that negative interactions mediated by the $\mathrm{HCN}$ operon of $P$. aeruginosa or cyanide in co-cultures with $B$. cenocepacia are rather complex. In fact, we show that the susceptibility of Burkholderia to cyanide is not only culture mediumdependent, but also reduced or even blocked via heat labile molecule(s) when non-toxic cyanide-resistant bacteria or subinhibitory concentrations of their metabolites are present within the community. Furthermore, within a population of $P$. aeruginosa from a single patient sample we observed a spectrum of interactions by doing pairwise co-cultures where cyanide production was not associated with killing, reinforcing the fact that cyanide is necessary, but not sufficient for killing and that extracellular factors mainly RhlR-dependent (Figure 2A) are required with cyanide for effective Burkholderia killing in vitro. Altogether, these demonstrate the complexity of interspecies competition and the unlimited numbers of possible interactions that may directly influence microbial diversity in the community. This work paves the way for future studies to dissect the role of $P$. aeruginosa-mediated competition in the establishment and development of chronic mixed infections with Burkholderia. Understanding these competitive interactions at the molecular level and their impact in vivo as well as how Burkholderia is more tolerant in chemically defined media will be essential for the implementation of new strategies for the treatment and management of lower airway polymicrobial infections.

\section{AUTHOR CONTRIBUTIONS}

SB designed the study, performed experiments, and wrote the manuscript. MW performed the HCN experiment on the $P$. aeruginosa clinical isolates. XL extracted and purified the rhamnolipids. NM and GO helped in the design of the study. MS designed the study and wrote the manuscript.

\section{ACKNOWLEDGMENTS}

We would like to thank Pamela A. Sokol and Deborah A. Hogan for generously providing some of the bacterial strains used in this study. We also want to thank Laura Rossi and Lori Burrows for critical reading of the manuscript. This study was supported by funding from Cystic Fibrosis Canada to MS. SB was the recipient of a postdoctoral research fellowship from Cystic Fibrosis Canada and MS is Canada Research Chair in Interdisciplinary Microbiome Research. GO is supported by NIH grant R37-AI83256.

\section{SUPPLEMENTARY MATERIAL}

The Supplementary Material for this article can be found online at: http://journal.frontiersin.org/article/10.3389/fmicb. 2016.00725 


\section{REFERENCES}

Al-Bakri, A. G., Gilbert, P., and Allison, D. G. (2004). Immigration and emigration of Burkholderia cepacia and Pseudomonas aeruginosa between and within mixed biofilm communities. J. Appl. Microbiol. 96, 455-463. doi: 10.1111/j.1365-2672.2004.02201.x

Anderson, R. D., Roddam, L. F., Bettiol, S., Sanderson, K., and Reid, D. W. (2010). Biosignificance of bacterial cyanogenesis in the CF lung. J. Cyst. Fibros. 9, 158-164. doi: 10.1016/j.jcf.2009.12.003

Avison, M. B., von Heldreich, C. J., Higgins, C. S., Bennett, P. M., and Walsh, T. R. (2000). A TEM-2beta-lactamase encoded on an active Tn1-like transposon in the genome of a clinical isolate of Stenotrophomonas maltophilia. J. Antimicrob. Chemother. 46, 879-884. doi: 10.1093/jac/46.6.879

Bakkal, S., Robinson, S. M., Ordonez, C. L., Waltz, D. A., and Riley, M. A. (2010). Role of bacteriocins in mediating interactions of bacterial isolates taken from cystic fibrosis patients. Microbiology 156, 2058-2067. doi: 10.1099/mic.0.036848-0

Bernier, S. P., Nguyen, D. T., and Sokol, P. A. (2008). A LysR-type transcriptional regulator in Burkholderia cenocepacia influences colony morphology and virulence. Infect. Immun. 76, 38-47. doi: 10.1128/IAI.00874-07

Bernier, S. P., and Surette, M. G. (2013). Concentration-dependent activity of antibiotics in natural environments. Front. Microbiol. 4:20. doi: 10.3389/fmicb. 2013.00020

Blumer, C., and Haas, D. (2000). Mechanism, regulation, and ecological role of bacterial cyanide biosynthesis. Arch. Microbiol. 173, 170-177. doi: 10.1007/s002039900127

Bragonzi, A., Farulla, I., Paroni, M., Twomey, K. B., Pirone, L., Lorè, N. I., et al. (2012). Modelling co-infection of the cystic fibrosis lung by Pseudomonas aeruginosa and Burkholderia cenocepacia reveals influences on biofilm formation and host response. PLOS ONE 7:e52330. doi: 10.1371/journal.pone.0052330

Caiazza, N. C., Shanks, R. M., and O’Toole, G. A. (2005). Rhamnolipids modulate swarming motility patterns of Pseudomonas aeruginosa. J. Bacteriol. 187, 7351-7361. doi: 10.1128/JB.187.21.7351-7361.2005

Carlier, A., Agnoli, K., Pessi, G., Suppiger, A., Jenul, C., Schmid, N., et al. (2014). Genome sequence of Burkholderia cenocepacia H111, a cystic fibrosis airway isolate. Genome Announc. 2:e00298-14. doi: 10.1128/genomeA.00298-14

Carmody, L. A., Zhao, J., Kalikin, L. M., Lebar, W., Simon, R. H., Venkataraman, A., et al. (2015). The daily dynamics of cystic fibrosis airway microbiota during clinical stability and at exacerbation. Microbiome 3:12. doi: 10.1186/s40168015-0074-9

Cassat, J., Dunman, P. M., Murphy, E., Projan, S. J., Beenken, K. E., Palm, K. J., et al. (2006). Transcriptional profiling of a Staphylococcus aureus clinical isolate and its isogenic agr and sarA mutants reveals global differences in comparison to the laboratory strain RN6390. Microbiology 152, 3075-3090. doi: 10.1099/mic.0.29033-0

Castric, K. F., and Castric, P. A. (1983). Method for rapid detection of cyanogenic bacteria. Appl. Environ. Microbiol. 45, 701-702.

Cipollone, R., Ascenzi, P., and Visca, P. (2007a). Common themes and variations in the rhodanese superfamily. IUBMB Life 59, 51-59. doi: $10.1080 / 15216540701206859$

Cipollone, R., Frangipani, E., Tiburzi, F., Imperi, F., Ascenzi, P., and Visca, P. (2007b). Involvement of Pseudomonas aeruginosa rhodanese in protection from cyanide toxicity. Appl. Environ. Microbiol. 73, 390-398. doi: 10.1128/AEM.02143-06

Coenye, T., Lipuma, J. J., Henry, D., Hoste, B., Vandemeulebroecke, K., Gillis, M., et al. (2001). Burkholderia cepacia genomovar VI, a new member of the Burkholderia cepacia complex isolated from cystic fibrosis patients. Int. J. Syst. Evol. Microbiol. 51, 271-279. doi: 10.1099/00207713-51-2-271

Coenye, T., Vandamme, P., Lipuma, J. J., Govan, J. R., and Mahenthiralingam, E. (2003). Updated version of the Burkholderia cepacia complex experimental strain panel. J. Clin. Microbiol. 41, 2797-2798. doi: 10.1128/jcm.41.6.27972798.2003

Cooper, M., Tavankar, G. R., and Williams, H. D. (2003). Regulation of expression of the cyanide-insensitive terminal oxidase in Pseudomonas aeruginosa. Microbiology 149, 1275-1284. doi: 10.1099/mic.0.26017-0

Costello, A., Reen, F. J., O’gara, F., Callaghan, M., and McClean, S. (2014). Inhibition of co-colonizing cystic fibrosis-associated pathogens by
Pseudomonas aeruginosa and Burkholderia multivorans. Microbiology 160, 1474-1487. doi: 10.1099/mic.0.074203-0

Cugini, C., Morales, D. K., and Hogan, D. A. (2010). Candida albicansproduced farnesol stimulates Pseudomonas quinolone signal production in LasR-defective Pseudomonas aeruginosa strains. Microbiology 156, 3096-3107. doi: $10.1099 /$ mic.0.037911-0

Cullen, L., and McClean, S. (2015). Bacterial adaptation during chronic respiratory infections. Pathogens 4, 66-89. doi: 10.3390/pathogens4010066

Cunningham, L., and Williams, H. D. (1995). Isolation and characterization of mutants defective in the cyanide-insensitive respiratory pathway of Pseudomonas aeruginosa. J. Bacteriol. 177, 432-438.

Curtis, T. P., Sloan, W. T., and Scannell, J. W. (2002). Estimating prokaryotic diversity and its limits. Proc. Natl. Acad. Sci. U.S.A. 99, 10494-10499. doi: 10.1073/pnas.142680199

Deziel, E., Lépine, F., Milot, S., He, J., Mindrinos, M. N., Tompkins, R. G., et al. (2004). Analysis of Pseudomonas aeruginosa 4-hydroxy-2alkylquinolines (HAQs) reveals a role for 4-hydroxy-2-heptylquinoline in cellto-cell communication. Proc. Natl. Acad. Sci. U.S.A. 101, 1339-1344. doi: 10.1073/pnas.0307694100

Dickson, R. P., Martinez, F. J., and Huffnagle, G. B. (2014). The role of the microbiome in exacerbations of chronic lung diseases. Lancet 384, 691-702. doi: 10.1016/S0140-6736(14)61136-3

Duan, K., Dammel, C., Stein, J., Rabin, H., and Surette, M. G. (2003). Modulation of Pseudomonas aeruginosa gene expression by host microflora through interspecies communication. Mol. Microbiol. 50, 1477-1491. doi: 10.1046/j.1365-2958.2003.03803.x

Filkins, L. M., Graber, J. A., Olson, D. G., Dolben, E. L., Lynd, L. R., Bhuju, S., et al. (2015). Coculture of Staphylococcus aureus with Pseudomonas aeruginosa drives $S$. aureus towards fermentative metabolism and reduced viability in a cystic fibrosis model. J. Bacteriol. 197, 2252-2264. doi: 10.1128/JB. 00059-15

Fridman, O., Goldberg, A., Ronin, I., Shoresh, N., and Balaban, N. Q. (2014). Optimization of lag time underlies antibiotic tolerance in evolved bacterial populations. Nature 513, 418-421. doi: 10.1038/nature13469

Gibson, J., Sood, A., and Hogan, D. A. (2009). Pseudomonas aeruginosa-Candida albicans interactions: localization and fungal toxicity of a phenazine derivative. Appl. Environ. Microbiol. 75, 504-513. doi: 10.1128/AEM.01037-08

Gilchrist, F. J., Sims, H., Alcock, A., Jones, A. M., Bright-Thomas, R. J., Smith, D., et al. (2013). Is hydrogen cyanide a marker of Burkholderia cepacia complex? J. Clin. Microbiol. 51, 3849-3851. doi: 10.1128/JCM.02157-13

Ha, D. G., Merritt, J. H., Hampton, T. H., Hodgkinson, J. T., Janecek, M., Spring, D. R., et al. (2011). 2-Heptyl-4-quinolone, a precursor of the Pseudomonas quinolone signal molecule, modulates swarming motility in Pseudomonas aeruginosa. J. Bacteriol. 193, 6770-6780. doi: 10.1128/JB. 05929-11

Hacek, D. M., Dressel, D. C., and Peterson, L. R. (1999). Highly reproducible bactericidal activity test results by using a modified National Committee for Clinical Laboratory Standards broth macrodilution technique. J. Clin. Microbiol. 37, 1881-1884.

Hall-Stoodley, L., Costerton, J. W., and Stoodley, P. (2004). Bacterial biofilms: from the natural environment to infectious diseases. Nat. Rev. Microbiol. 2, 95-108. doi: 10.1038/nrmicro821

Han, M. K., Huang, Y. J., Lipuma, J. J., Boushey, H. A., Boucher, R. C., Cookson, W. O., et al. (2012). Significance of the microbiome in obstructive lung disease. Thorax 67, 456-463. doi: 10.1136/thoraxjnl-2011-201183

Harrison, F. (2007). Microbial ecology of the cystic fibrosis lung. Microbiology 153, 917-923. doi: 10.1099/mic.0.2006/004077-0

Hoffman, L. R., Déziel, E., D’Argenio, D. A., Lêpine, F., Emerson, J., McNamara, S., et al. (2006). Selection for Staphylococcus aureus small-colony variants due to growth in the presence of Pseudomonas aeruginosa. Proc. Natl. Acad. Sci. U.S.A. 103, 19890-19895. doi: 10.1073/pnas.0606756104

Hoffman, L. R., Kulasekara, H. D., Emerson, J., Houston, L. S., Burns, J. L., Ramsey, B. W., et al. (2009). Pseudomonas aeruginosa lasR mutants are associated with cystic fibrosis lung disease progression. J. Cyst. Fibros. 8, 66-70. doi: 10.1016/j.jcf.2008.09.006

Hogan, D. A., and Kolter, R. (2002). Pseudomonas-Candida interactions: an ecological role for virulence factors. Science 296, 2229-2232. doi: $10.1126 /$ science. 1070784 
Hogan, D. A., Vik, A., and Kolter, R. (2004). A Pseudomonas aeruginosa quorumsensing molecule influences Candida albicans morphology. Mol. Microbiol. 54, 1212-1223. doi: 10.1111/j.1365-2958.2004.04349.x

Holden, M. T., Seth-Smith, H. M., Crossman, L. C., Sebaihia, M., Bentley, S. D., Cerdeño-Tárraga, A. M., et al. (2009). The genome of Burkholderia cenocepacia J2315, an epidemic pathogen of cystic fibrosis patients. J. Bacteriol. 191, 261-277. doi: 10.1128/JB.01230-08

Huang, Y. J., and Lynch, S. V. (2011). The emerging relationship between the airway microbiota and chronic respiratory disease: clinical implications. Expert Rev. Respir. Med. 5, 809-821. doi: 10.1586/ers.11.76

Huber, B., Riedel, K., Hentzer, M., Heydorn, A., Gotschlich, A., Givskov, M., et al. (2001). The cep quorum-sensing system of Burkholderia cepacia H111 controls biofilm formation and swarming motility. Microbiology 147, 2517-2528. doi: 10.1099/00221287-147-9-2517

Jacques, I., Derelle, J., Weber, M., and Vidailhet, M. (1998). Pulmonary evolution of cystic fibrosis patients colonized by Pseudomonas aeruginosa and/or Burkholderia cepacia. Eur. J. Pediatr. 157, 427-431. doi: 10.1007/s004310050844

Jimenez, P. N., Koch, G., Thompson, J. A., Xavier, K. B., Cool, R. H., and Quax, W. J. (2012). The multiple signaling systems regulating virulence in Pseudomonas aeruginosa. Microbiol. Mol. Biol. Rev. 76, 46-65. doi: 10.1128/MMBR.05007-11

Jorth, P., Staudinger, B. J., Wu, X., Hisert, K. B., Hayden, H., Garudathri, J., et al. (2015). Regional isolation drives bacterial diversification within cystic fibrosis lungs. Cell Host Microbe 18, 307-319. doi: 10.1016/j.chom.2015.07.006

Kelsic, E. D., Zhao, J., Vetsigian, K., and Kishony, R. (2015). Counteraction of antibiotic production and degradation stabilizes microbial communities. Nature 521, 516-519. doi: 10.1038/nature14485

Kerr, B., Riley, M. A., Feldman, M. W., and Bohannan, B. J. (2002). Local dispersal promotes biodiversity in a real-life game of rock-paper-scissors. Nature 418, 171-174. doi: 10.1038/nature00823

Korgaonkar, A., Trivedi, U., Rumbaugh, K. P., and Whiteley, M. (2013). Community surveillance enhances Pseudomonas aeruginosa virulence during polymicrobial infection. Proc. Natl. Acad. Sci. U.S.A. 110, 1059-1064. doi: 10.1073/pnas.1214550110

Lambiase, A., Raia, V., Del Pezzo, M., Sepe, A., Carnovale, V., and Rossano, F. (2006). Microbiology of airway disease in a cohort of patients with cystic fibrosis. BMC Infect. Dis. 6:4. doi: 10.1186/1471-2334-6-4

Lenney, W., and Gilchrist, F. J. (2011). Pseudomonas aeruginosa and cyanide production. Eur. Respir. J. 37, 482-483. doi: 10.1183/09031936.00122810

Liberati, N. T., Urbach, J. M., Miyata, S., Lee, D. G., Drenkard, E., Wu, G., et al. (2006). An ordered, nonredundant library of Pseudomonas aeruginosa strain PA14 transposon insertion mutants. Proc. Natl. Acad. Sci. U.S.A. 103, 2833-2838. doi: 10.1073/pnas.0511100103

Lipuma, J. J. (2010). The changing microbial epidemiology in cystic fibrosis. Clin. Microbiol. Rev. 23, 299-323. doi: 10.1128/CMR.00068-09

Lipuma, J. J., Spilker, T., Coenye, T., and Gonzalez, C. F. (2002). An epidemic Burkholderia cepacia complex strain identified in soil. Lancet 359, 2002-2003. doi: 10.1016/S0140-6736(02)08836-0

Mahenthiralingam, E., Coenye, T., Chung, J. W., Speert, D. P., Govan, J. R., Taylor, P., et al. (2000). Diagnostically and experimentally useful panel of strains from the Burkholderia cepacia complex. J. Clin. Microbiol. 38, 910-913.

McKeon, S. A., Nguyen, D. T., Viteri, D. F., Zlosnik, J. E., and Sokol, P. A. (2011). Functional quorum sensing systems are maintained during chronic Burkholderia cepacia complex infections in patients with cystic fibrosis. J. Infect. Dis. 203, 383-392. doi: 10.1093/infdis/jiq054

Mowat, E., Paterson, S., Fothergill, J. L., Wright, E. A., Ledson, M. J., Walshaw, M. J., et al. (2011). Pseudomonas aeruginosa population diversity and turnover in cystic fibrosis chronic infections. Am. J. Respir. Crit. Care Med. 183, 1674-1679. doi: 10.1164/rccm.201009-1430OC

Murray, J. L., Connell, J. L., Stacy, A., Turner, K. H., and Whiteley, M. (2014). Mechanisms of synergy in polymicrobial infections. J. Microbiol. 52, 188-199. doi: 10.1007/s12275-014-4067-3

Nair, C., Shoemark, A., Chan, M., Ollosson, S., Dixon, M., Hogg, C., et al. (2014). Cyanide levels found in infected cystic fibrosis sputum inhibit airway ciliary function. Eur. Respir. J. 44, 1253-1261. doi: 10.1183/09031936.00097014

Neerincx, A. H., Mandon, J., Van Ingen, J., Arslanov, D. D., Mouton, J. W., Harren, F. J., et al. (2015). Real-time monitoring of hydrogen cyanide (HCN) and ammonia (NH3) emitted by Pseudomonas aeruginosa. J. Breath Res. 9:027102. doi: $10.1088 / 1752-7155 / 9 / 2 / 027102$
Odds, F. C. (2003). Synergy, antagonism, and what the chequerboard puts between them. J. Antimicrob. Chemother. 52:1. doi: 10.1093/jac/dkg301

Palmer, K. L., Aye, L. M., and Whiteley, M. (2007). Nutritional cues control Pseudomonas aeruginosa multicellular behavior in cystic fibrosis sputum. J. Bacteriol. 189, 8079-8087. doi: 10.1128/JB.01138-07

Park, S. Y., Heo, Y. J., Choi, Y. S., Deziel, E., and Cho, Y. H. (2005). Conserved virulence factors of Pseudomonas aeruginosa are required for killing Bacillus subtilis. J. Microbiol. 43, 443-450.

Parkins, M. D., Glezerson, B. A., Sibley, C. D., Sibley, K. A., Duong, J., Purighalla, S., et al. (2014). Twenty-five-year outbreak of Pseudomonas aeruginosa infecting individuals with cystic fibrosis: identification of the prairie epidemic strain. $J$. Clin. Microbiol. 52, 1127-1135. doi: 10.1128/JCM.03218-13

Pasteur, L., and Joubert, J. (1877). Charbon et septicémie. Compt. Rend. Acad. 85, 101-105.

Peters, B. M., Jabra-Rizk, M. A., O’May, G. A., Costerton, J. W., and Shirtliff, M. E. (2012). Polymicrobial interactions: impact on pathogenesis and human disease. Clin. Microbiol. Rev. 25, 193-213. doi: 10.1128/CMR.00013-11

Pillai, S. K., Moellering, R. C., and Eliopoulos, G. M. (2005). “Antimicrobial combinations," in Antibiotics in Laboratory Medicine, 5th Edn., ed V. Lorian. (Philadelphia, PA: The Lippincott Williams \& Wilkins Co.), 365-440.

Pinzon, N. M., and Ju, L. K. (2009). Improved detection of rhamnolipid production using agar plates containing methylene blue and cetyl trimethylammonium bromide. Biotechnol. Lett. 31, 1583-1588. doi: 10.1007/s10529-009-0049-7

Price-Whelan, A., Dietrich, L. E., and Newman, D. K. (2006). Rethinking 'secondary' metabolism: physiological roles for phenazine antibiotics. Nat. Chem. Biol. 2, 71-78. doi: 10.1038/nchembio764

Pukatzki, S., Kessin, R. H., and Mekalanos, J. J. (2002). The human pathogen Pseudomonas aeruginosa utilizes conserved virulence pathways to infect the social amoeba Dictyostelium discoideum. Proc. Natl. Acad. Sci. U.S.A. 99, 3159-3164. doi: 10.1073/pnas.052704399

Rahim, R., Ochsner, U. A., Olvera, C., Graninger, M., Messner, P., Lam, J. S., et al. (2001). Cloning and functional characterization of the Pseudomonas aeruginosa rhlC gene that encodes rhamnosyltransferase 2, an enzyme responsible for di-rhamnolipid biosynthesis. Mol. Microbiol. 40, 708-718. doi: 10.1046/j.13652958.2001.02420.x

Rahme, L. G., Stevens, E. J., Wolfort, S. F., Shao, J., Tompkins, R. G., and Ausubel, F. M. (1995). Common virulence factors for bacterial pathogenicity in plants and animals. Science 268, 1899-1902. doi: 10.1126/science.7604262

Rüger, M., Ackermann, M., and Reichl, U. (2014). Species-specific viability analysis of Pseudomonas aeruginosa, Burkholderia cepacia and Staphylococcus aureus in mixed culture by flow cytometry. BMC Microbiol. 14:56. doi: 10.1186/14712180-14-56

Ryall, B., Lee, X., Zlosnik, J. E., Hoshino, S., and Williams, H. D. (2008). Bacteria of the Burkholderia cepacia complex are cyanogenic under biofilm and colonial growth conditions. BMC Microbiol. 8:108. doi: 10.1186/1471-2180-8-108

Schwab, U., Abdullah, L. H., Perlmutt, O. S., Albert, D., Davis, C. W., Arnold, R. R., et al. (2014). Localization of Burkholderia cepacia complex bacteria in cystic fibrosis lungs and interactions with Pseudomonas aeruginosa in hypoxic mucus. Infect. Immun. 82, 4729-4745. doi: 10.1128/IAI.01876-14

Shanks, R. M., Caiazza, N. C., Hinsa, S. M., Toutain, C. M., and O’Toole, G. A. (2006). Saccharomyces cerevisiae-based molecular tool kit for manipulation of genes from gram-negative bacteria. Appl. Environ. Microbiol. 72, 5027-5036. doi: 10.1128/AEM.00682-06

Short, F. L., Murdoch, S. L., and Ryan, R. P. (2014). Polybacterial human disease: the ills of social networking. Trends Microbiol. 22, 508-516. doi: 10.1016/j.tim.2014.05.007

Sibley, C. D., Duan, K., Fischer, C., Parkins, M. D., Storey, D. G., Rabin, H. R., et al. (2008). Discerning the complexity of community interactions using a Drosophila model of polymicrobial infections. PLoS Pathog. 4:e1000184. doi: 10.1371/journal.ppat.1000184

Smalley, N. E., An, D., Parsek, M. R., Chandler, J. R., and Dandekar, A. A. (2015). Quorum sensing protects Pseudomonas aeruginosa against cheating by other species in a laboratory coculture model. J. Bacteriol. 197, 3154-3159. doi: 10.1128/JB.00482-15

Smith, E. E., Buckley, D. G., Wu, Z., Saenphimmachak, C., Hoffman, L. R., D’Argenio, D. A., et al. (2006). Genetic adaptation by Pseudomonas aeruginosa to the airways of cystic fibrosis patients. Proc. Natl. Acad. Sci. U.S.A. 103, 8487-8492. doi: 10.1073/pnas.0602138103 
Surette, M. G. (2014). The cystic fibrosis lung microbiome. Ann. Am. Thorac. Soc. 11(suppl. 1), S61-S65. doi: 10.1513/annalsats.201306-159mg

Tashiro, Y., Yawata, Y., Toyofuku, M., Uchiyama, H., and Nomura, N. (2013). Interspecies interaction between Pseudomonas aeruginosa and other microorganisms. Microbes Environ. 28, 13-24. doi: 10.1264/jsme2. ME12167

Tomlin, K. L., Coll, O. P., and Ceri, H. (2001). Interspecies biofilms of Pseudomonas aeruginosa and Burkholderia cepacia. Can. J. Microbiol. 47, 949-954. doi: 10.1139/w01-095

Tyrrell, J., Whelan, N., Wright, C., Sá-Correia, I., McClean, S., Thomas, M., et al. (2015). Investigation of the multifaceted iron acquisition strategies of Burkholderia cenocepacia. Biometals 28, 367-380. doi: 10.1007/s10534-0159840-1

Varga, J. J., Losada, L., Zelazny, A. M., Kim, M., McCorrison, J., Brinkac, L., et al. (2013). Draft Genome sequences of Burkholderia cenocepacia ET12 lineage strains K56-2 and BC7. Genome Announc. 1:e00841-13. doi: 10.1128/genomeA.00841-13

Wang, M., Schaefer, A. L., Dandekar, A. A., and Greenberg, E. P. (2015). Quorum sensing and policing of Pseudomonas aeruginosa social cheaters. Proc. Natl. Acad. Sci. U.S.A. 112, 2187-2191. doi: 10.1073/pnas.1500 704112

Weaver, V. B., and Kolter, R. (2004). Burkholderia spp. alter Pseudomonas aeruginosa physiology through iron sequestration. J. Bacteriol. 186, 2376-2384. doi: 10.1128/JB.186.8.2376-2384.2004
Workentine, M. L., Sibley, C. D., Glezerson, B., Purighalla, S., Norgaard-Gron, J. C., Parkins, M. D., et al. (2013). Phenotypic heterogeneity of Pseudomonas aeruginosa populations in a cystic fibrosis patient. PLoS ONE 8:e60225. doi: 10.1371/journal.pone.0060225

Workentine, M. L., Surette, M. G., and Bernier, S. P. (2014). Draft genome sequence of Burkholderia dolosa PC543 isolated from cystic fibrosis airways. Genome Announc. 2:e00043-14. doi: 10.1128/genomeA.00043-14

Wybouw, N., Dermauw, W., Tirry, L., Stevens, C., Grbic, M., Feyereisen, R., et al. (2014). A gene horizontally transferred from bacteria protects arthropods from host plant cyanide poisoning. Elife 3:e02365. doi: 10.7554/eLife.02365

Zdor, R. E. (2015). Bacterial cyanogenesis: impact on biotic interactions. J. Appl. Microbiol. 118, 267-274. doi: 10.1111/jam.12697

Conflict of Interest Statement: The authors declare that the research was conducted in the absence of any commercial or financial relationships that could be construed as a potential conflict of interest.

Copyright (C) 2016 Bernier, Workentine, Li, Magarvey, O’Toole and Surette. This is an open-access article distributed under the terms of the Creative Commons Attribution License (CC BY). The use, distribution or reproduction in other forums is permitted, provided the original author(s) or licensor are credited and that the original publication in this journal is cited, in accordance with accepted academic practice. No use, distribution or reproduction is permitted which does not comply with these terms. 